\title{
Efeitos da eletroestimulação na função musculoventilatória em humanos: revisão de escopo
}

\author{
Effects of electrical stimulation on musculoventilatory \\ function in humans: scope review
}

\author{
Efectos de la estimulación eléctrica sobre la función \\ musculoventilatoria en humanos: revisión del alcance
}

\author{
Maria Itamara da Silva Oliveira ${ }^{1}$, Rafaela Pedrosa ${ }^{2}$, Tatiana Onofre \\ Gama ${ }^{3}$, José Heriston de Morais Lima ${ }^{4}$, Ubiraçé Fernando Elihimas \\ Júnior $^{5}$, Suellen Marinho Andrade ${ }^{6}$, Eduardo Eriko Tenório de França ${ }^{7}$
}

\begin{abstract}
1.Fisioterapia, Discente do curso de graduação de Fisioterapia, Universidade Federal da Paraíba, Campus João Pessoa, João Pessoa-PB, Brasil. ORCID: https://orcid.org/0000-0002-5022-2862

2.Professora adjunta, Doutora, Universidade Federal da Paraíba, Departamento de Fisioterapia, Campus João Pessoa, João Pessoa-PB, Brasil. ORCID: https://orcid.org/0000-0001-9858-2990

3.Professora adjunta, Doutora, Universidade Federal da Paraíba, Departamento de Fisioterapia, Campus João Pessoa, João Pessoa-PB, Brasil. ORCID: https://orcid.org/0000-0003-4985-1466

4.Professor adjunto, Pós-doutor, Universidade Federal da Paraíba, Departamento de Fisioterapia, Campus João Pessoa, João Pessoa-PB, Brasil. ORCID: https://orcid.org/0000-0002-0176-6222

5.Médico, Doutor, Universidade de Pernambuco, Departamento de Ciências da Saúde, Campus Recife, Recife-PE, Brasil. ORCID: https://orcid.org/000-0001-5426-4253

6.Professora adjunta, Doutor, Universidade Federal da Paraíba, Departamento de Fisioterapia e Programa de Pós-graduação em Fisioterapia, Campus João Pessoa, João Pessoa-PB, Brasil. ORCID: https://orcid.org/0000-0002-6801-0462

7.Professor adjunto, Doutor, Universidade Federal da Paraíba, Departamento de Fisioterapia e Programa de Pós-graduação em Fisioterapia, Campus João Pessoa, João Pessoa-PB, Brasil. ORCID: https://orcid.org/0000-0001-9207-2180
\end{abstract}

\begin{abstract}
Resumo
Introdução. Várias técnicas de neuromodulação vêm sendo utilizadas com grande potencial terapêutico e a eletroestimulação tem surgido na literatura científica e na prática clínica com finalidades e resultados surpreendentes. Objetivo. Analisar os efeitos das técnicas de eletroestimulação transcraniana por corrente contínua (ETCC) e estimulação elétrica transcutânea (EET) na função musculoventilatória em seres humanos. Método. Trata-se de uma revisão de escopo com pesquisa bibliográfica realizada nas bases de dados BVS e PubMed, utilizando-se como referência o checklist PRIMA-Scr. Resultados. Foram recuperados 282 estudos e após o processo de elegibilidade, 15 estudos foram analisados, destes, sete avaliaram os efeitos da ETCC e oito, os da EET. A ETCC foi aplicada sob diferentes protocolos experimentais, majoritariamente em apenas uma sessão de estimulação, por 10 ou 20 minutos, em $1 \mathrm{~mA}$ ou $2 \mathrm{~mA}$, e com montagem de eletrodos variáveis, conforme área cerebral estimulada. Seus efeitos foram heterogêneos, variando de melhora à um desfecho negativo. Já a EET abrangeu diferentes correntes elétricas e processos metodológicas. A maioria utilizou uma sessão de estimulação, variando de 15 a 240 minutos, intensidade de corrente e posicionamento de eletrodos variável, dependente do tipo de corrente e da área estimulada. Em todos houve melhora de pelo menos uma variável de estudo. Conclusão. Os resultados da EET se mostraram satisfatórios melhora da função musculoventilatória de seres humanos. Já os da ETCC se revelaram ainda pouco elucidados, requerendo estudos mais homogêneos. Sugere-se a realização de mais pesquisas para sanar lacunas sobre os efeitos dessas correntes, a curto e longo prazo.
\end{abstract}

Unitermos. Eletroestimulação transcraniana por corrente contínua; estimulação elétrica transcutânea; sistema nervoso autônomo; diafragma; Respiração 


\begin{abstract}
Introduction. Several neuromodulation techniques have been used with great therapeutic potential and electrostimulation has emerged in the scientific literature and clinical practice with surprising purposes and results. Objectives. To analyze the effects of transcranial direct current electrical stimulation (tDCS) and transcutaneous electrical stimulation (TSE) techniques on musculoventilatory function in humans. Method. This is a scope review with bibliographic research carried out in the BVS and PubMed databases, using the PRIMA-Scr checklist as a reference. Results. 282 studies were retrieved and after the eligibility process, 15 studies were analyzed, of which seven evaluated the effects of tDCS and eight, those of TSE. tDCS was applied under different experimental protocols, mostly in just one stimulation session, for 10 or 20 minutes, in $1 \mathrm{~mA}$ or $2 \mathrm{~mA}$, and with variable electrode assembly, according to the stimulated brain area. Its effects were heterogeneous, ranging from improvement to a negative outcome. The TSE, on the other hand, covered different electrical currents and methodological processes. Most used a stimulation session, ranging from 15 to 240 minutes, current intensity and variable electrode placement, depending on the type of current and the stimulated area. In all, there was an improvement in at least one study variable. Conclusions. The TSE results showed satisfactory improvement in the musculoventilatory function of human beings. The SDCS, on the other hand, proved to be poorly understood, requiring more homogeneous studies. It is suggested that more research be carried out to remedy gaps in the effects of these currents, in the short and long term.
\end{abstract}

Keywords. Transcranial direct current electrostimulation; transcutaneous electrical stimulation; Autonomic nervous system; diaphragm; Breathing

\begin{abstract}
Resumen
Introducción. Se han utilizado varias técnicas de neuromodulación con gran potencial terapéutico y la electroestimulación ha surgido en la literatura científica y en la práctica clínica con propósitos y resultados sorprendentes. Método. Analizar los efectos de las técnicas de estimulación eléctrica transcraneal de corriente continua (ETCC) y estimulación eléctrica transcutánea (EET) sobre la función musculoventilatoria en humanos. Método. Se trata de una revisión de alcance con investigación bibliográfica realizada en las bases de datos BVS y PubMed, utilizando como referencia el checklist PRIMA-Scr. Resultados. Se recuperaron 282 estudios y luego del proceso de elegibilidad, se analizaron 15 estudios, de los cuales siete evaluaron los efectos de ETCC y ocho, los de EET. La ETCC se aplicó bajo diferentes protocolos experimentales, la mayoría en una sola sesión de estimulación, durante 10 o 20 minutos, en $1 \mathrm{~mA}$ o $2 \mathrm{~mA}$, y con montaje de electrodos variable, según el área cerebral estimulada. Sus efectos fueron heterogéneos, desde una mejoría hasta un resultado negativo. El EET, por su parte, cubrió diferentes corrientes eléctricas y procesos metodológicos. La mayoría utilizó una sesión de estimulación, que oscilaba entre 15 y 240 minutos, intensidad de corriente y colocación de electrodos variable, según el tipo de corriente y la zona estimulada. En total, hubo una mejora en al menos una variable de estudio. Conclusiones. Los resultados de la EET mostraron una mejora satisfactoria en la función musculoventilatoria de los seres humanos. EI ETCC, por otro lado, demostró estar poco esclarecido, requiriendo estudios más homogéneos. Se sugiere que se realicen más investigaciones para subsanar las lagunas en los efectos de estas corrientes, a corto y largo plazo.
\end{abstract}

Palabras clave. Electroestimulación transcraneal de corriente continua; estimulación eléctrica transcutánea; Sistema nervioso autónomo; diafragma; Respiración

Trabalho realizado para conclusão de curso de graduação em Fisioterapia da Universidade Federal da Paraíba, Campus João Pessoa, João Pessoa-PB, Brasil.

Conflito de interesse: não

Recebido em: 18/08/2021

Aceito em: $28 / 10 / 2021$

Endereço de correspondência: Eduardo Eriko Tenório de França. Universidade Federal da Paraíba. Conj. Pres. Castelo Branco III, João Pessoa-PB, Brasil. CEP 58033-455. Fone +55 (83) 32167183. E-mail: edueriko@hotmail.com 


\section{INTRODUÇÃO}

Nas últimas décadas, várias técnicas de neuromodulação vêm sendo utilizadas com grande potencial terapêutico. A eletroestimulação transcraniana por corrente contínua (ETCC) e a estimulação elétrica transcutânea (EET) tem surgido na literatura científica e na prática clínica com finalidades e resultados surpreendentes.

A ETCC faz uso de técnica experimental não-invasiva, indolor e de fácil aplicação, na qual o estímulo de baixa intensidade de corrente anódica promove aumento da excitabilidade cortical enquanto o estímulo de corrente catódica apresenta efeito oposto ${ }^{1}$. Estudos em humanos apontam que a modulação da excitabilidade cortical durante a estimulação com ETCC ocorre por mudanças não sinápticas nas células, todavia há evidências de que os efeitos mais tardios do uso da corrente são devidos a modificações sinápticas ${ }^{2}$.

Existem atualmente vários estudos com a aplicação da ETCC em áreas específicas do córtex motor, inclusive com efeitos na respiração. Autores mostraram que a estimulação magnética transcraniana repetitiva (EMTr) a $5 \mathrm{~Hz}$ aplicada na área motora suplementar (AMS) aumentou simultaneamente a amplitude do potencial evocado motor (PEM) diafragmático e dos primeiros músculos interósseos dorsais, sugerindo que a modificação da excitabilidade da AMS induz modificações da excitabilidade da área do córtex motor primário, e consequentemente do córtex motor diafragmático ${ }^{3}$. Embora os respectivos mecanismos EMTr e ETCC sejam diferentes, 
esses resultados podem sugerir que a ETCC aplicada no córtex motor primário do diafragma, por exemplo, pode modificar sua excitabilidade. Apesar disso, ainda há limitadas informações sobre os benefícios potenciais da ETCC sobre aspectos ventilatórios dos indivíduos.

Em se tratando da EET, tem sido descrito alguns de seus efeitos sobre a função musculoventilatória humana, como por exemplo, a estimulação diafragmática elétrica transcutânea (EDET) e sua ação sobre a funcionalidade do diafragma. A EDET utiliza-se de estímulos elétricos rítmicos de curta duração e eletrodos de superfície para gerar um potencial de ação frênico, estimulando a contração diafragmática e, consequentemente, promovendo uma pressão intrapleural negativa que pode influenciar na ventilação pulmonar ${ }^{4}$.

Além desta, outras aplicações da EET têm sido desenvolvidas com a finalidade da melhora da função ventilatória, tais como: a aplicação trans-espinhal, sobre músculos abdominais, sobre pontos de acupuntura, entre outros $^{5-7}$. Nos casos de pacientes com disfunções da musculatura inspiratória, como dos músculos intercostais e abdominais, a ação da EET sobre a musculatura disfuncional resultaria em potencialização dessa ação muscular e consequente aumento da capacidade ventilatória. No entanto, são escassos os estudos que avaliam a eficácia desses métodos.

Apesar do amplo espectro de possibilidades da ação efetiva da estimulação elétrica sobre a fisiologia 
neuromuscular, são raros os estudos que descrevem seus resultados sobre a função musculoventilatória da população humana. Sendo assim, surge a necessidade do agrupamento e da análise revisional de estudos que retratem essa temática.

Esse estudo trata-se, então, de uma revisão de escopo que objetiva analisar as contribuições de pesquisas nacionais e internacionais a respeito dos efeitos das técnicas de estimulação elétrica, seja ela transcraniana ou transcutânea, na função musculoventilatória em seres humanos, além de identificar lacunas de pesquisa para a elaboração de estudos futuros.

\section{MÉTODO}

Trata-se de uma revisão de escopo que tem se destacado mundialmente na área de síntese de evidências em saúde, comumente utilizada para examinar a extensão, o alcance e a natureza da atividade de pesquisa em uma área temática. Nesta revisão utilizou-se como referência o guia para relatório de revisão de escopo Preferred Reporting Items for Systematic reviews and Meta-Analyses extension for Scoping Reviews (PRISMA-ScR) Checklist ${ }^{8}$. De acordo com os requisitos indicados pelo PRISMA-ScR, a pergunta norteadora da pesquisa foi elaborada tendo como elementoschave a população ou participantes, conceitos e contexto. Sendo assim, o estudo foi orientado pela seguinte pergunta: 
"O que a literatura científica refere sobre os efeitos da ETCC e da EET na função musculoventilatória em humanos?".

Foram incluídos nesta revisão documentos que atenderam os seguintes critérios: formato de artigo, com resultados que abordaram os efeitos da estimulação elétrica na função musculoventilatória de seres humanos. Foram excluídos artigos de revisão e documentos não completamente disponíveis nas bases de dados ou na internet. Tendo em vista que não há outras revisões de literatura com essa temática, não foi utilizado o filtro para delimitação do ano de publicação.

Foi realizada pesquisa bibliográfica nas bases de dados eletrônicas da Biblioteca Virtual de Saúde (BVS) e PubMed, reconhecendo estudos que abordavam a relação ou procedimento do uso da ETCC ou EET sobre a função musculoventilatória em humanos. Para tal, utilizou-se termos de busca nas línguas portuguesa (BVS) e inglesa (PubMed), recuperando-se em uma busca geral o conteúdo de publicações. Foi utilizado o conector booleano "AND" para combinar os termos sugeridos, definida em busca nos "Títulos e Resumos", com filtro de acesso aberto aos artigos. As buscas nas duas bases de dados foram realizadas nos dias 07 (PubMed) e 08 (BVS) de março de 2021 (Tabela 1 e Tabela 2). 
Tabela 1. Termos de busca e número de manuscritos recuperados na base de dados BIREME/BVS.

\begin{tabular}{|c|c|}
\hline \multirow{2}{*}{ TERMOS DE BUSCA } & BASE DE DADOS \\
\cline { 2 - 2 } & BIREME/BVS \\
\hline Estimulação Transcraniana por Corrente Contínua "and" Diafragma & $\mathrm{n}=0$ \\
\hline Estimulação Transcraniana por Corrente Contínua "and" Ventilação & $\mathrm{n}=1$ \\
\hline Estimulação Transcraniana por Corrente Contínua "and" Respiração & $\mathrm{n}=4$ \\
\hline Estimulação Transcraniana por Corrente Contínua "and" Pulmão & $\mathrm{n}=0$ \\
\hline Estimulação Transcraniana por Corrente Contínua "and" Exercícios Respiratórios & $\mathrm{n}=1$ \\
\hline Estimulação Transcraniana por Corrente Contínua "and" Músculos Respiratórios & $\mathrm{n}=1$ \\
\hline Estimulação Transcraniana por Corrente Contínua "and" Sistema Nervoso Autônomo & $\mathrm{n}=7$ \\
\hline Estimulação Elétrica Transcutânea "and" Diafragma & $\mathrm{n}=42$ \\
\hline Estimulação Elétrica Transcutânea "and" Músculos Respiratórios & $\mathrm{n}=18$ \\
\hline Estimulação Elétrica Transcutânea "and" Exercícios Respiratórios & $\mathrm{n}=7$ \\
\hline Estimulação Elétrica Transcutânea "and" Ventilação & $\mathrm{n}=15$ \\
\hline Estimulação Elétrica Transcutânea "and" Respiração & $\mathrm{n}=41$ \\
\hline Estimulação Elétrica Transcutânea "and" Pulmão & $\mathrm{n}=42$ \\
\hline Estimulação Elétrica Transcutânea "and" Sistema Nervoso Autônomo & $\mathrm{n}=26$ \\
\hline
\end{tabular}

BVS= Biblioteca Virtual de Saúde.

Tabela 2. Termos de busca e número de manuscritos recuperados na base de dados PubMed.

\begin{tabular}{|c|c|}
\hline TERMOS DE BUSCA & BASE DE DADOS \\
\cline { 2 - 2 } & PubMed \\
\hline Transcranial Direct Current Stimulation "and" Diaphragm & $\mathrm{n}=1$ \\
\hline Transcranial Direct Current Stimulation "and" Ventilation & $\mathrm{n}=4$ \\
\hline Transcranial Direct Current Stimulation "and" Respiration & $\mathrm{n}=8$ \\
\hline Transcranial Direct Current Stimulation "and" lung & $\mathrm{n}=4$ \\
\hline Transcranial Direct Current Stimulation "and" Breathing Exercises & $\mathrm{n}=1$ \\
\hline Transcranial Direct Current Stimulation "and" respiratory muscles & $\mathrm{n}=1$ \\
\hline Transcranial Direct Current Stimulation "and" Autonomic Nervous System & $\mathrm{n}=18$ \\
\hline Transcutaneous electrical nerve stimulation "and" Diaphragm & $\mathrm{n}=1$ \\
\hline Transcutaneous electrical nerve stimulation "and" Ventilation & $\mathrm{n}=7$ \\
\hline Transcutaneous electrical nerve stimulation "and" Respiration & $\mathrm{n}=1$ \\
\hline Transcutaneous electrical nerve stimulation "and" respiratory muscles & $\mathrm{n}=1$ \\
\hline Transcutaneous electrical nerve stimulation "and" lung & $\mathrm{n}=15$ \\
\hline Transcutaneous electrical nerve stimulation "and" Breathing Exercises & $\mathrm{n}=2$ \\
\hline Transcutaneous electrical nerve stimulation "and" Autonomic Nervous System & $\mathrm{n}=13$ \\
\hline
\end{tabular}

PubMed = Serviço de publicações da U.S. National Library of Medicine. 
Diante disso, a seleção final foi feita da seguinte forma: inicialmente, (1) houve a exclusão de documentos que não fossem artigos e não correspondessem aos critérios de inclusão exigidos anteriormente, (2) foram excluídos artigos repetidos. Em seguida, foram aceitos artigos potencialmente elegíveis a partir da (3) leitura dos títulos, (4) leitura dos objetivos, (5) leitura dos resultados e (6) leitura dos textos completos. Com isso, foi realizado uma síntese qualitativa (narrativa) das evidências encontradas em resposta à pergunta da pesquisa.

\section{RESULTADOS}

\section{Características amostral dos estudos incluídos}

Através da pesquisa bibliográfica inicial que combinou termos apresentados nas Tabelas 1 e 2, foram recuperados 282 estudos, sendo 205 encontrados na BVS e 77 no PubMed, dos quais 80 eram duplicados em ambas as bases de dados e foram, portanto, excluídos. Foram rastreados 202 artigos, sendo que destes, foram excluídos 181 após a leitura dos títulos e resumos e 6 após a leitura dos textos completos, restando 15 estudos para análise. O processo de identificação, seleção, elegibilidade e inclusão dos estudos podem ser observados na Figura 1. 
Figura 1: Fluxo de identificação, seleção e inclusão das produções científicas.

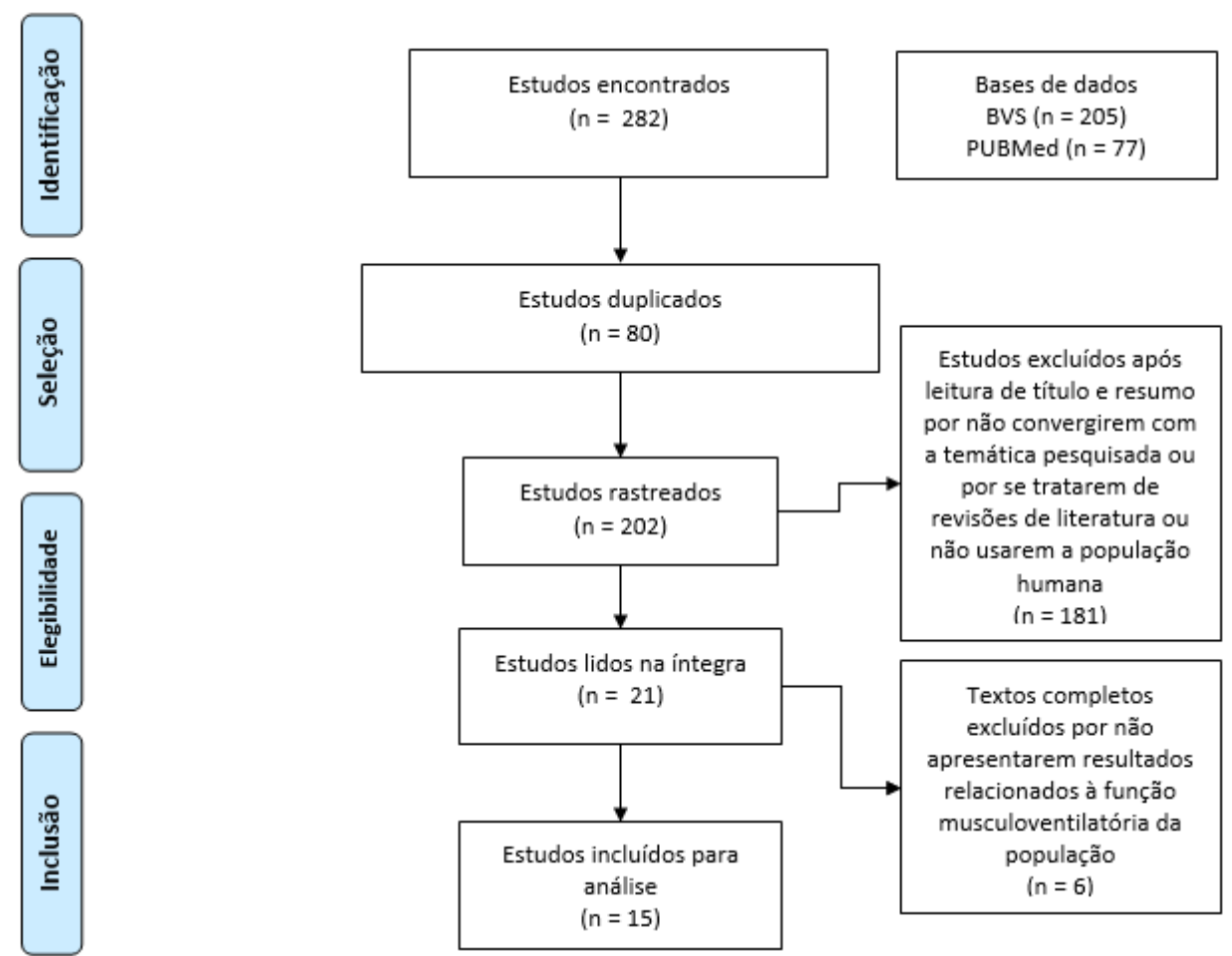

BVS= Biblioteca Virtual de Saúde; PubMed= Serviço de publicações da U.S. National Library of Medicine.

A Tabela 3 descreve uma síntese dos estudos selecionados para esta revisão segundo autor, tipo de estimulação, metodologia, posição de eletrodos, variáveis de interesse e principais desfechos de cada estudo.

\section{Descrição dos estudos}

Sete estudos avaliaram os efeitos da ETCC $^{9-15}$ e oito estudos os resultados da EET ${ }^{5-7,16-20}$. Dentre os estudos com EET, um estudo utilizou a Acu-TENS (Estimulação elétrica nervosa transcutânea acunpuntura)7; outros dois, a TENS (Estimulação elétrica nervosa trancutânea) ${ }^{16,17}$; três, a EDET ${ }^{18-20 ; ~ u m, ~ a ~ A F E S ~(E s t i m u l a c ̧ a ̃ o ~ e l e ́ t r i c a ~ f u n c i o n a l ~}$ 
abdominal) ${ }^{6}$; e outro, a EteCC (Estimulação transespinhal

por corrente contínua) ${ }^{5}$. Os artigos relacionados

apresentaram uma variação de 10 a 46 participantes,

somando um total de 315 participantes estudados.

Tabela 3. Síntese dos estudos incluídos na revisão segundo autor, tipo de estimulação, metodologia, posição de eletrodos, variáveis de interesse e principais desfechos de cada estudo.

\begin{tabular}{|c|c|c|c|c|c|c|}
\hline Autor & $\begin{array}{c}\text { Tipo de } \\
\text { estimulação }\end{array}$ & Grupo & Metodologia & $\begin{array}{l}\text { Posição dos } \\
\text { eletrodos }\end{array}$ & $\begin{array}{c}\text { Variáveis de } \\
\text { interesse }\end{array}$ & Conclusão \\
\hline $\begin{array}{l}\text { Vandermeeren; } \\
\text { et al., } 2010^{9}\end{array}$ & ETCC & $\begin{array}{c}\text { N total }=30 \\
\text { N A/ETCC }=10 \\
\text { N C/ETCC }=10 \\
\text { N Sham }=10\end{array}$ & $\begin{array}{c}\text { Estudo exploratório } \\
\text { simples-cego, } \\
\text { controlado por sham } \\
\text { Indivíduos saudáveis } \\
\text { A/ETCC e } \\
\text { C/ETCC =duração de } \\
20 \text { min, } 1 \mathrm{~mA} \text {, fade } \\
\text { in/out } 8 \mathrm{~s} \text {. } \\
\text { Sham = duração de } \\
30 \mathrm{~s}, 1 \mathrm{~mA} \text {, fade } \\
\text { in/out } 8 \mathrm{~s}, 14 \mathrm{~s} \\
\text { ativado }\end{array}$ & $\begin{array}{l}\text { Eletrodo ativo na } \\
\text { linha média sobre Fz } \\
\text { e o eletrodo de } \\
\text { referência } \\
\text { extracefálico sobre a } \\
\text { tíbia direita }\end{array}$ & FR & $\begin{array}{c}\text { S/ alterações } \\
\text { significantes entre } \\
\text { grupos }\end{array}$ \\
\hline $\begin{array}{c}\text { Azabou et al., } \\
2013^{10}\end{array}$ & ETCC & $\begin{array}{c}\mathrm{N} \text { total }=12 \\
\text { Todos } \\
\text { passaram } \\
\text { pelos três } \\
\text { procedimentos }\end{array}$ & $\begin{array}{c}\text { Ensaio clínico } \\
\text { Homens saudáveis } \\
\text { A/ETCC ou C/ETCC= } \\
\text { intensidade de } 2 \\
\mathrm{~mA} \text {; duração de } 10 \\
\text { min; densidade de } \\
\text { corrente de } 0,057 \\
\mathrm{~mA} / \mathrm{cm} 2 \\
\text { Sham }=\text { intensidade } \\
\text { de } 2 \mathrm{~mA} \text {; duração de } \\
2 \text { min }\end{array}$ & $\begin{array}{c}\text { A/ETCC }=\text { ânodo } \\
\text { sobre o córtex motor } \\
\text { primário } \\
\text { diafragmático } \\
\text { esquerdo }(4 \mathrm{~cm} \\
\text { lateral à linha média } \\
\text { e } 1 \mathrm{~cm} \text { anterior à } \\
\text { linha binaural) e } \\
\text { cátodo acima da } \\
\text { órbita direita. } \\
\text { C/ETCC=colocação } \\
\text { contrária à A/ETCC } \\
\end{array}$ & $\begin{array}{l}\text { LM } \\
\text { PEM }\end{array}$ & $\begin{array}{c}\text { Sham }=\text { LM; PEM s/ } \\
\text { alt. } \\
\text { A/ETCC }=\text { LM s/ } \\
\text { alt.; PEM } \downarrow \\
\text { C/ETCC }=\text { LM s/ } \\
\text { alt.; } \\
\text { PEM } \downarrow\end{array}$ \\
\hline $\begin{array}{c}\text { Tomczak et al., } \\
2013^{11}\end{array}$ & ETCC & $\begin{array}{c}\mathrm{N} \text { total }=10 \\
\mathrm{~N} \text { A/ETCC }=\text { não } \\
\text { informado } \\
\mathrm{N} \text { C/ETCC=não } \\
\text { informado } \\
\mathrm{N} \text { sham =não } \\
\text { informado }\end{array}$ & $\begin{array}{c}\text { Estudo randomizado, } \\
\text { cego simples } \\
\text { Indivíduos saudáveis } \\
\text { Sham=aumento do } \\
\text { estimulador ao longo } \\
\text { de } 10 \mathrm{~s} \text { e } \\
\text { manutenção da } \\
\text { estimulação por } 10 \mathrm{~s} \\
\text { a uma meta de } 1 \mathrm{~mA} \\
\text { A/ETCC e C/ETCC= } \\
\text { aumento de } 10 \mathrm{~s} \\
\text { para } 1 \mathrm{~mA} \text {, seguido } \\
\text { por } 10 \text { min de } \\
\text { estimulação a } 1 \mathrm{~mA}\end{array}$ & $\begin{array}{l}\text { Sobre a região do } \\
\text { córtex motor } \\
\text { esquerdo e região } \\
\text { supraorbital } \\
\text { contralateral. }\end{array}$ & $\begin{array}{l}\text { Coerência } \\
\text { intermuscular } \\
\text { Cinemática da } \\
\text { parede torácica } \\
\text { (V início e } \\
\text { término, } \\
\text { excursão do } \\
\text { volume pulmonar } \\
\text { total) }\end{array}$ & $\begin{array}{c}\text { A/ETCC=área de } \\
\text { coerência } \\
\text { intermuscular } \uparrow \text { na } \\
\mathrm{CV} \text {, cinemática da } \\
\text { parede torácica s/ } \\
\text { alt. } \\
\text { Sham = área de } \\
\text { coerência e } \\
\text { cinemática da } \\
\text { parede torácica s/ } \\
\text { alt. } \\
\text { C/ETCC= área de } \\
\text { coerência e } \\
\text { cinemática da } \\
\text { parede torácica s/ } \\
\text { alt. }\end{array}$ \\
\hline
\end{tabular}


Tabela 4 (cont.). Síntese dos estudos incluídos na revisão segundo autor, tipo de estimulação, metodologia, posição de eletrodos, variáveis de interesse e principais desfechos de cada estudo.

\begin{tabular}{|c|c|c|c|c|c|c|}
\hline Autor & $\begin{array}{c}\text { Tipo de } \\
\text { estimulação }\end{array}$ & Grupo & Metodologia & $\begin{array}{l}\text { Posição dos } \\
\text { eletrodos }\end{array}$ & $\begin{array}{c}\text { Variáveis de } \\
\text { interesse }\end{array}$ & Conclusão \\
\hline $\begin{array}{c}\text { Montenegro et } \\
\text { al., } 2014^{12}\end{array}$ & ETCC & $\begin{array}{c}\text { N total }=11 \\
\text { N Sham=não } \\
\text { informado N } \\
\text { A/ETCC= não } \\
\text { informado }\end{array}$ & $\begin{array}{c}\text { Ensaio clínico } \\
\text { Homens saudáveis } \\
\text { tDCS sham ou } \\
\text { anodal, } 2 \mathrm{~mA}, 20 \\
\text { min, e } 30 \text { minutos } \\
\text { após o exercício } \\
\text { aeróbio isocalórico }\end{array}$ & $\begin{array}{l}\text { Ânodo sobre a área } \\
\text { F3 e eletrodo de } \\
\text { referência sobre a } \\
\text { área supraorbital } \\
\text { contralateral (Fp2). } \\
\text { Para sham, o } \\
\text { estimulador foi } \\
\text { desligado após } 30 \mathrm{~s}\end{array}$ & VO2 & $\begin{array}{c}\text { DURANTE =s/ alt. } \\
\text { significantes entre } \\
\text { grupos } \\
\text { PÓS } 30 \text { MIN = } \\
\text { A/ETCC VO2 } \uparrow \text { em } \\
\text { comparação com } \\
\text { Sham }\end{array}$ \\
\hline $\begin{array}{l}\text { Lee et al., } \\
2017^{13}\end{array}$ & ETCC & $\begin{array}{c}\text { N total }=30 \\
\text { N A/ETCC }=15 \\
\text { N Sham }=15\end{array}$ & $\begin{array}{c}\text { Estudo randomizado } \\
\text { Pacientes com AVC } \\
\text { A/ETCC=exercícios } \\
\text { de respiração } \\
\text { diafragmática + } 20 \\
\text { min estimulação } \\
\text { Sham=exercícios de } \\
\text { respiração } \\
\text { diafragmática + } 20 \\
\text { min estimulação } \\
\text { (dispositivo foi } \\
\text { desligado } 30 \\
\text { segundos depois) } \\
\end{array}$ & $\begin{array}{c}\text { O ânodo sobre a } \\
\text { área C3 e C4 } \\
\text { (sistema } \\
\text { internacional 10-20 } \\
\text { EEG) e o cátodo foi } \\
\text { colocado na área } \\
\text { supraorbital do outro } \\
\text { lado }\end{array}$ & $\begin{array}{l}\text { CVF } \\
\mathrm{VEF}_{1}\end{array}$ & $\begin{array}{c}\mathrm{A} / \mathrm{ETCC}=\mathrm{CVF} \uparrow \uparrow, \\
\mathrm{VEF}_{1} \uparrow \uparrow \\
\text { Sham }=\mathrm{CVF} \uparrow, \\
\operatorname{VEF}_{1} \uparrow\end{array}$ \\
\hline $\begin{array}{c}\text { Baldari et al., } \\
2018^{14}\end{array}$ & ETCC & $\begin{array}{c}\mathrm{N} \text { total }=13 \\
\text { Todos } \\
\text { passaram pelos } \\
\text { três } \\
\text { experimentos }\end{array}$ & $\begin{array}{c}\text { Estudo duplo-cego, } \\
\text { randomizado e } \\
\text { contrabalançado } \\
\text { Corredores } \\
\text { saudáveis } \\
\text { A/ETCC e } \\
\text { C/ETCC=Corrente de } \\
2 \text { mA, duração de } 20 \\
\text { min com rampa de } \\
10 \text { s no início e no } \\
\text { final } \\
\text { Sham = } 1 \text { mA por } \\
30 \mathrm{~s} \text {, com uma } \\
\text { rampa de } 20 \mathrm{~s}\end{array}$ & $\begin{array}{l}\text { Um eletrodo sobre a } \\
\text { região } \mathrm{Cz}(\sim 4,5 \mathrm{~cm} \\
\text { de cada lado do } \\
\text { córtex motor } \\
\text { primário), e o outro } \\
\text { sobre a } \\
\text { protuberância } \\
\text { occipital }\end{array}$ & $\begin{array}{l}\text { AEP } \\
\mathrm{VO}_{2} \\
\mathrm{VE} \\
\text { GET }\end{array}$ & $\begin{array}{c}\text { S/ alterações } \\
\text { significantes entre } \\
\text { as condições }\end{array}$ \\
\hline $\begin{array}{l}\text { Park et al., } \\
2019^{15}\end{array}$ & ETCC & $\begin{array}{c}\mathrm{N} \text { total }=10 \\
\text { Todos } \\
\text { passaram pela } \\
\text { sham e } \mathrm{A} / \mathrm{ETCC}\end{array}$ & $\begin{array}{l}\text { Estudo randomizado, } \\
\text { simples-cego e } \\
\text { contrabalançado } \\
\text { Corredores treinados } \\
\text { A/ETCC }=1,98 \mathrm{~mA} \text {, } \\
\text { duração de } 20 \text { min e } \\
\text { em seguida corrida } \\
\text { em } 80 \% \text { VO2máx } \\
\text { Sham }=\text { corrente por } \\
\text { 30s e depois } \\
\text { desligada }\end{array}$ & $\begin{array}{c}\text { Os ânodos em CZ } \\
\text { (linha média central) } \\
\text { no topo do 'vértice' } \\
\text { da cabeça, e os } \\
\text { catódos em C5 e C6 } \\
\text { (sistema } \\
\text { internacional 10-20 } \\
\text { EEG) }\end{array}$ & $\begin{array}{l}\mathrm{VO}_{2} \\
\mathrm{VE} \\
\mathrm{QR} \\
\mathrm{LV}\end{array}$ & $\begin{array}{c}\text { A/ETCC e sham= } \\
\text { S/ alterações } \\
\text { significantes }\end{array}$ \\
\hline $\begin{array}{l}\text { Cipriano Jr et } \\
\text { al., } 2008^{16}\end{array}$ & EET/TENS & $\begin{array}{c}\mathrm{N} \text { total }=45 \\
\mathrm{~N} \text { TENS } \\
\text { terapêutica }=23 \\
\mathrm{~N} \text { TENS } \\
\text { placebo }=22\end{array}$ & $\begin{array}{c}\text { Estudo prospectivo e } \\
\text { randomizado. } \\
\text { Pacientes pós } \\
\text { cirurgia cardíaca } \\
\text { TENS } \\
\text { terapêutica }=\text { frequên } \\
\text { cia de onda de } 80 \mathrm{~Hz} \\
\text { e um ciclo de } \\
\text { trabalho de } 330 / 33 \\
\text { ms, duração de } 240 \\
\text { min. } \\
\text { TENS placebo= } \\
\text { tempo de descanso } \\
\text { entre os pulsos de } \\
350 \text { ms para } 330 \mathrm{~s} \text {. }\end{array}$ & $\begin{array}{c}\text { Sobre a região } \\
\text { subclavicular de } \\
\text { cada lado da incisão, } \\
\text { aproximadamente } \\
2-3 \mathrm{~cm} \text { da linha de } \\
\text { sutura. }\end{array}$ & $\begin{array}{l}\text { CV } \\
\text { VC } \\
\text { FR }\end{array}$ & $\begin{array}{c}\text { TENS } \\
\text { terapêutica }=\mathrm{CV} \uparrow, \\
\text { VC } \uparrow, \mathrm{FR} \downarrow \\
\text { TENS placebo }=\mathrm{CV} \\
\uparrow, \text { VC } \uparrow, \mathrm{FR} \text { s/ } \\
\text { alteração }\end{array}$ \\
\hline
\end{tabular}


Tabela 5 (cont.). Síntese dos estudos incluídos na revisão segundo autor, tipo de estimulação, metodologia, posição de eletrodos, variáveis de interesse e principais desfechos de cada estudo.

\begin{tabular}{|c|c|c|c|c|c|c|}
\hline Autor & $\begin{array}{c}\text { Tipo de } \\
\text { estimulação }\end{array}$ & Grupo & Metodologia & $\begin{array}{l}\text { Posição dos } \\
\text { eletrodos }\end{array}$ & $\begin{array}{l}\text { Variáveis de } \\
\text { interesse }\end{array}$ & Conclusão \\
\hline $\begin{array}{l}\text { Lau et al., } \\
\qquad 2008^{7}\end{array}$ & $\begin{array}{l}\text { EET/Acu- } \\
\text { TENS }\end{array}$ & $\begin{array}{c}\mathrm{N} \text { total }=46 \\
\mathrm{~N} \\
\text { experimental }= \\
23 \\
\mathrm{~N} \text { controle }=23\end{array}$ & $\begin{array}{c}\text { Estudo randomizado } \\
\text { controlado por } \\
\text { placebo } \\
\text { Pacientes com DPOC } \\
\text { estágio I ou II; } \\
\text { Experimental=4Hz, } \\
\text { 200ms, } 45 \text { min. de } \\
\text { Acu-TENS; } \\
\text { Controle= }=\text { placebo } \\
\text { Acu-TENS }\end{array}$ & $\begin{array}{c}\text { Experimental= } \\
\text { acuponto Ex-B1 } \\
\text { bilateralmente }(0,5 \\
\text { 'cun' lateral ao } \\
\text { processo espinhoso } \\
\text { da } 7 \text { a vértebra } \\
\text { cervical) } \\
\text { Controle= idêntica, } \\
\text { mas sem saída } \\
\text { elétrica. }\end{array}$ & $\begin{array}{c}\mathrm{VEF}_{1} \\
\text { CVF } \\
\text { Dispneia }\end{array}$ & $\begin{array}{c}\text { Experimental }=V F_{1} \\
\uparrow \text { em } 0,12 \text { litros e } \\
\text { dispneia } \downarrow \text { em } 10,7 \\
\text { mm mais do que o } \\
\text { controle. } \\
\text { CVF com diferença } \\
\text { não significante }\end{array}$ \\
\hline $\begin{array}{c}\text { Gregorini et al., } \\
2010^{17}\end{array}$ & EET/TENS & $\begin{array}{c}\mathrm{N} \text { total }=25 \\
\mathrm{~N} \text { TENS } \\
\text { terapêutica }=13 \\
\mathrm{~N} \text { TENS } \\
\text { placebo }=12\end{array}$ & $\begin{array}{c}\text { Ensaio clínico } \\
\text { prospectivo e } \\
\text { randomizado } \\
\text { Pacientes pós } \\
\text { cirurgia cardíaca } \\
\text { TENS } \\
\text { terapêutica =taxa de } \\
\text { pulso de } 80 \mathrm{~Hz} \text {, } \\
\text { largura de pulso de } \\
150 \mu \mathrm{s} \text {, duração de } \\
240 \text { min. } \\
\text { TENS } \\
\text { placebo=tempo } \\
\text { ativo entre os pulsos } \\
\text { de } 330 \\
\text { milissegundos para } \\
33 \text { segundos }\end{array}$ & $\begin{array}{l}\text { Um eletrodo de cada } \\
\text { lado da ferida } \\
\text { operatória na região } \\
\text { subclávia, } \\
\text { aproximadamente } \\
\text { dois a três } \mathrm{cm}\end{array}$ & $\begin{array}{l}\text { PImáx } \\
\text { PEmáx } \\
\text { VC } \\
\text { CV } \\
\text { FR } \\
\text { VE }\end{array}$ & $\begin{array}{l}\text { TENS terapêutica }= \\
\text { PImáx } \uparrow, P E m a ́ x \\
\uparrow, V C \uparrow, C V \uparrow \uparrow, F R \\
\text { S/ alt, VE } \uparrow \uparrow \\
\text { TENS placebo= } \\
\text { PImáx } \downarrow, P E m a ́ x \text { S/ } \\
\text { alt., VC } \downarrow, C V \uparrow, F R \\
\text { S/ alt., VE } \uparrow\end{array}$ \\
\hline $\begin{array}{c}\text { Cancelliero- } \\
\text { Gaiad et al., } \\
2012^{18}\end{array}$ & EET/EDET & $\begin{array}{c}N \text { total }=21 \\
N \text { Controle } \\
(n=7) \\
\text { EDET com } \\
\text { Phrenics }(n=7) \\
\text { EDET com } \\
\text { Dualpex }(n=7)\end{array}$ & $\begin{array}{l}\text { Estudo controlado e } \\
\text { randomizado } \\
\text { Mulheres saudáveis } \\
2 \text { vezes por semana, } \\
\text { durante } 6 \text { semanas } \\
\text { EDET Phrenics= } \\
\text { corrente pulsada, } \\
\text { bifásica e simétrica, } \\
\text { frequência de } 30 \mathrm{~Hz} \text {; } \\
\text { largura de pulso de } \\
0,4 \mathrm{~ms} \text {, subida de } \\
0,7 \mathrm{~s} \text { e FR de } 15 \\
\text { rpm, sustentação de } \\
0,4 \mathrm{~s} \text {, duração de } 30 \\
\text { min } \\
\text { EDET Dualpex= } \\
\text { corrente pulsada, } \\
\text { bifásica e simétrica, } \\
\text { frequência de } 30 \mathrm{~Hz} \text {; } \\
\text { largura de pulso de } \\
0,4 \text { ms; rampa de } 1 \\
\text { s; sustentação de } 1 \\
\text { s e descida de } 2 \mathrm{~s} \text {. } \\
\text { Controle=sem } \\
\text { intervenção }\end{array}$ & $\begin{array}{l}\text { Um eletrodo na } \\
\text { região paraxifoidea } \\
\text { direita e esquerda } \\
\text { no terceiro espaço } \\
\text { intercostal e o outro } \\
\text { na linha axilar } \\
\text { média, na região do } \\
\text { sétimo espaço } \\
\text { intercostal }\end{array}$ & $\begin{array}{l}\text { PImáx } \\
\text { PEmáx }\end{array}$ & $\begin{array}{c}\text { EDET } \\
\text { Dualpex }=\text { PImáx } \\
\uparrow \uparrow, \text { PEmáx } \uparrow \uparrow \\
\text { EDET } \\
\text { Phrenics }=\text { PImáx } \uparrow \text {, } \\
\text { Pemáx } \uparrow \\
\text { Controle }=\mathrm{s} / \text { alt. }\end{array}$ \\
\hline $\begin{array}{c}\text { Cancelliero- } \\
\text { Gaiad et al., } \\
2013^{19}\end{array}$ & EET/ETED & $\begin{array}{c}\text { N total }=15 \\
\mathrm{~N} \\
\text { Respondente }=9 \\
\mathrm{~N} \text { Não } \\
\text { Respondente }=6\end{array}$ & $\begin{array}{c}\text { Ensaio clínico } \\
\text { transversal, } \\
\text { comparativo e não } \\
\text { randomizado } \\
\text { Pacientes com DPOC } \\
\text { Corrente pulsada, } \\
\text { bifásica e simétrica: } \\
\text { frequência de } 30 \\
\text { hertz; duração do } \\
\text { pulso de } 0,4 \text { ms; } \\
\text { tempo de subida de } \\
0,7 \text { s; duração de } 20 \\
\text { min. }\end{array}$ & $\begin{array}{l}\text { Em cada lado do } \\
\text { tórax, no } 3^{\circ} \text { espaço } \\
\text { intercostal próximo } \\
\text { à região xifóide e no } \\
70 \text { espaço } \\
\text { intercostal, na linha } \\
\text { axilar média. }\end{array}$ & $\begin{array}{l}\text { VE } \\
\text { VCinp } \\
\text { VCexp } \\
\text { FR } \\
\text { SatO }_{2}\end{array}$ & $\begin{array}{c}\text { Respondente }=\text { VM } \\
\uparrow ; \text { VCinsp } \uparrow ; \text { VCexp } \\
\uparrow ; \text { FR s/ alt.; } \\
\text { SatO2 } \uparrow \\
\text { Não Respondente= } \\
\text { VM } \downarrow \text {; VCinsp } \downarrow ; \\
\text { VCexp } \downarrow ; \text { FR s/ alt. }\end{array}$ \\
\hline
\end{tabular}


Tabela 6 (cont.). Síntese dos estudos incluídos na revisão segundo autor, tipo de estimulação, metodologia, posição de eletrodos, variáveis de interesse e principais desfechos de cada estudo.

\begin{tabular}{|c|c|c|c|c|c|c|}
\hline Autor & $\begin{array}{c}\text { Tipo de } \\
\text { estimulação }\end{array}$ & Grupo & Metodologia & $\begin{array}{l}\text { Posição dos } \\
\text { eletrodos }\end{array}$ & $\begin{array}{l}\text { Variáveis de } \\
\text { interesse }\end{array}$ & Conclusão \\
\hline $\begin{array}{c}\text { Mclachlan et } \\
\text { al., } 2013^{6}\end{array}$ & EET/AFES & $\mathrm{N}$ total $=12$ & $\begin{array}{c}\text { Estudo longitudinal } \\
\text { Pacientes com lesão } \\
\text { medular tetraplégica } \\
\text { Fase de controle } \\
\text { (semana } 1 \text { ) } \\
\text { Fase de treinamento } \\
\text { (semanas } 2-4 \text { )= } \\
\text { corrente bifásica a } \\
30 \mathrm{~Hz} \text {; } 4 \text { /semana; } \\
20 \mathrm{~min} / \text { dia para } 60 \\
\text { min/dia } \\
\text { Fase de } \\
\text { acompanhamento } \\
\text { (semanas 5-7) } \\
\end{array}$ & $\begin{array}{l}\text { Sobre o músculo } \\
\text { reto abdominal e os } \\
\text { grupos de músculos } \\
\text { oblíquos externos. }\end{array}$ & $\begin{array}{c}\text { CVF } \\
\text { VEF }_{1} \\
\text { FEP } \\
\text { PEmáx } \\
\text { VEF }_{1} / \text { CVF }\end{array}$ & $\begin{array}{c}\text { Fase de controle= } \\
\text { s/ alt. significantes } \\
\text { Fase de } \\
\text { treinamento=CVF } \\
\uparrow ; \mathrm{VEF}_{1} \uparrow ; \mathrm{PFE} \uparrow ; \\
\mathrm{PEmáx} \text { e } \mathrm{VEF} \mathrm{F}_{1} / \mathrm{CVF} \\
\text { s/ alt. significantes } \\
\text { Fase de } \\
\text { acompanhamento } \\
=\mathrm{s} / \text { alt. } \\
\text { significantes; PFE } \uparrow\end{array}$ \\
\hline $\begin{array}{c}\text { Niérat et al., } \\
2014^{5}\end{array}$ & EET/EteCC & $\begin{array}{c}\text { Conjunto } \\
\text { N total } 22 \\
\text { Conjunento } \\
1=\text { Experimento } \\
\text { principal } \\
(\mathrm{N}=17), \\
\text { controle } \mathrm{A} \\
(\mathrm{N}=7) \mathrm{e} \\
\text { controle } \mathrm{B} \\
(\mathrm{N}=7) \\
\text { Conjunto } \\
2=\text { Experimento } \\
\text { principal } \\
(\mathrm{N}=12) \mathrm{e} \\
\text { controle } \mathrm{C} \\
(\mathrm{N}=7)\end{array}$ & $\begin{array}{c}\text { Estudo duplo-cego, } \\
\text { controlado, } \\
\text { randomizado e } \\
\text { cruzado } \\
\text { Indivíduos saudáveis } \\
\text { Intensidade de } 2,5 \\
\text { mA; duração de } 15 \\
\text { min. } \\
\text { Conjunto } 1 \text { (efeitos } \\
\text { eletrofisiológicos)=E } \\
\text { xperimento principal } \\
\text { (A/EteCC, C/ETCC, } \\
\text { Sham; avaliou } \\
\text { PEMs), controle A } \\
\text { (A/EteCC, C/EteCC, } \\
\text { Sham; avaliou LMr e } \\
\text { SICI) e B (C/EteCC; } \\
\text { avaliou PAMCs) } \\
\text { Conjunto } 2 \text { (efeitos } \\
\text { ventilatórios)=Experi } \\
\text { mento principal } \\
\text { (A/EteCC, C/EteCC; } \\
\text { Sham; avaliou } \\
\text { padrão respiratório) } \\
\text { e controle C } \\
\text { (C/EteCC; avaliou } \\
\text { função autonômica) }\end{array}$ & $\begin{array}{c}\text { Um eletrodo sobre a } \\
\text { linha média da parte } \\
\text { posterior do pescoço } \\
\text { para cobrir os } \\
\text { segmentos espinhais } \\
\text { cervicais C3-C5, e o } \\
\text { outro sobre a linha } \\
\text { média da parte } \\
\text { anterior do pescoço, } \\
\text { logo abaixo do } \\
\text { ângulo } \\
\text { cervicomental }\end{array}$ & $\begin{array}{c}\text { PEM } \\
\text { diafragmático } \\
\text { LM } \\
\text { SICI } \\
\text { VC } \\
\text { VFC }\end{array}$ & $\begin{array}{c}\text { C/ETCC }= \\
\text { amplitude PEM } \uparrow ; \\
\text { LM } \downarrow ; \text { SICI e VCF } \\
\text { s/ alt.; VC } \uparrow \\
\text { A/ETCC }= \\
\text { amplitude PEM } \uparrow, \\
\text { LM } \downarrow \text {, SICI e VCF } \\
\text { s/ alt., VC s/ alt. } \\
\text { Sham = PEM, LM, } \\
\text { SICI, VC e VFC s/ } \\
\text { alt. }\end{array}$ \\
\hline $\begin{array}{l}\text { Santos et al., } \\
2015^{20}\end{array}$ & EET/EDET & $\mathrm{N}$ total $=13$ & $\begin{array}{c}\text { Estudo retrospectiva } \\
\text { quase- } \\
\text {-experimental } \\
\text { Pacientes com DPOC } \\
\text { grau III e IV } \\
\text { Corrente russa com t } \\
\text { e f de } 18 \mathrm{~min} \text {. ( } 20 \text { a } \\
30 \mathrm{~Hz} \text { ) e } 12 \text { min. (70 } \\
\text { a } 100 \mathrm{~Hz}), 1 \text {-6s de } \\
\text { contração e o tempo } \\
\text { de relaxamento } 2 \times 0 \\
\text { tempo de contração, } \\
30 \text { sessões } \\
\text { (2x/semana). }\end{array}$ & Não informado & $\begin{array}{c}\text { Dispneia } \\
\text { FR } \\
\text { Cirtometria } \\
\text { tóraco-abdominal }\end{array}$ & $\begin{array}{l}\text { Dispneia e FR s/ } \\
\text { alt. significantes; } \\
\text { cirtometria } \downarrow \text { axilar } \\
\text { expiratória, } \\
\text { torácica, torácica } \\
\text { inspiratória e } \\
\text { umbilical } \\
\text { expiratória }\end{array}$ \\
\hline
\end{tabular}

$\mathrm{N}=$ número; $\mathrm{ETCC}=$ estimulação transcraniana por corrente contínua; $\mathrm{A} / \mathrm{ETCC}=$ estimulação transcraniana por corrente contínua anodal; $C / E T C C=$ estimulação transcraniana por corrente contínua catodal; $E E T=e$ timulação elétrica transcutânea;TENS=estimulação elétrica nervosa transcutânea; Acu-TENS= estimulação elétrica nervosa transcutânea acupuntura; EtsCC=estimulação transespinhal por corrente contínua; EDET= estimulação diafragmática elétrica transcutânea; AFES=estimulação elétrica funcional abdominal; $\mathrm{mA}=$ miliampére; $\mathrm{Hz}=\mathrm{Hertz} ; \mathrm{rpm}=$ respirações por minuto; $\mathrm{ms}=$ milissegundos; $\mathrm{DPOC}=$ doença pulmonar obstrutiva crônica; $\mathrm{AVC}=$ acidente vascular cerebral; $\mathrm{PEM}=$ potencial evocado motor; $\mathrm{LMr}=$ limiar motor em repouso; SICI= inibição intracortical curta;

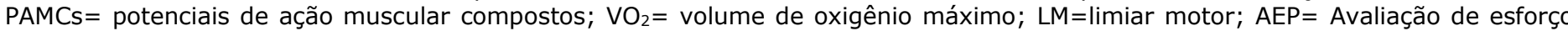
percebido; $V E=$ ventilação pulmonar; GET= limiar de troca gasosa; PImáx=pressão inspiratória máxima; PEmáx=pressão expiratória máxima; VCinp=volume corrente inspiratório; $V C e x p=$ volume corrente expiratório; $F R=$ frequência respiratória; SatO2=saturação periférica de oxigênio; $V C=$ capacidade vital; $V C=$ volume corrente; $V E F_{1}=$ volume expiratório forçado no primeiro segundo; $\mathrm{CFV}=$ capacidade vital forçada; $\mathrm{FEP}=$ fluxo expiratório de pico; $\mathrm{VFC}=$ variabilidade de frequência cardíaca; $\mathrm{QR}=$ razão de troca respiratória; LV=limiar ventilatório 
Metodologicamente, dos quinze estudos analisados, treze eram ensaios clínicos ${ }^{5,7,9-19}$ e outros dois artigos representavam um, um estudo retrospectivo quase experimental ${ }^{20}$, e o outro, um estudo longitudinal ${ }^{6}$. Em se tratanto dos tipos de condições experimentais de ETCC empregados em seus métodos, quatro estudos utilizaram três procedimentos distintos: sham, ETCC anodal (A/ETCC) e ETCC catodal (C/ETCC) $)^{9-11,14}$; e os outros três fizeram uso de apenas duas condições de estimulação diferentes: A/ETCC e sham ${ }^{12,13,15}$. Apenas um estudo analisou os efeitos da estimulação associado à outra intervenção ${ }^{13}$.

Em relação a EET, com exceção dos estudos de Mclachlan et al. ${ }^{6}$ e de Santos et al. ${ }^{20}$, todos os outros utilizaram grupos comparatórios em sua análise. Um estudo comparou os efeitos de dois diferentes protocolos de EDET sobre a força muscular respiratória de mulheres saudáveis ${ }^{18}$. Nos dois estudos que comparam os efeitos da TENS houve alocação dos participantes em um grupo TENS terapêutica e outro TENS placebo ${ }^{16,17}$. Cancelliero-Gaiad et al. ${ }^{19}$, em seu estudo com pacientes portadores da doença pulmonar obstrutiva crônica (DPOC), fizeram a alocação de pacientes após a estimulação elétrica, designando-os em um grupo "respondente" e outro "não respondente" à terapia.

Ademais, sobre o tempo utilizado para a intervenção, os estudos variaram entre uma e trinta sessões de estimulação. A maioria dos estudos utilizou apenas uma sessão para avaliar os efeitos da estimulação elétrica ${ }^{5,7,9-}$ $11,13,14,16,17,19$. Os estudos de Cancelliero-Gaiad et al. ${ }^{18} \mathrm{e}$ 
Mclachlan et al. ${ }^{6}$ utilizaram doze sessões. Outros dois estudos fizeram uso de duas sessões de estimulação em seus participantes ${ }^{12,15}$. Além disso, o estudo de Santos et al. ${ }^{20}$ utilizou o maior número de sessões de intervenção, trinta. $E$, com exceção de dois estudos ${ }^{12,15}$, todos os outros que aplicaram a ETCC estabeleceram seus resultados com 0 emprego de apenas uma sessão de estimulação elétrica.

Em se tratando da duração da estimulação, para os estudos com ETCC esta foi de 10 minutos $^{10,11}$ ou 20 minutos ${ }^{9,12-15}$. Já para os estudos com o uso da EET, houve variação de 15 a 240 minutos de estimulação. Destes estudos, um estimulou pacientes saudáveis por 15 minutos ${ }^{5}$, outro utilizou 20 minutos de estimulação ${ }^{19}$, dois estudos fizeram uso de 30 minutos para a intervenção ${ }^{18,20}$, um com duração de 45 minutos $^{7}$, e a estimulação com duração de 240 minutos foi realizada em dois estudos ${ }^{16,17}$. Um último artigo aumentou a duração da sessão por semana de intervenção: de 20 minutos na primeira semana a 60 minutos na terceira ${ }^{6}$.

Um outro aspecto que se destaca nos estudos é a densidade ou intensidade da corrente elétrica, a qual pode variar a depender do tipo de corrente elétrica utilizada. Nos estudos que abordaram a ETCC, a densidade da corrente esteve entre $1 \mathrm{~mA}$ e $2 \mathrm{~mA}$.

A intensidade da corrente entre os estudos com EET, diferentemente da ETCC, não era fixa, mas estabeleceramse dentro de alguns parâmetros fisiológicos de cada participante. Em alguns estudos essa intensidade foi 
ajustada como a mais alta tolerada pelo sujeito, com finalidade de permitir um forte formigamento ou forte contração muscular $6,7,16,17$. Já em outros estudos essa intensidade foi ajustada como a mínima a fim de permitir certa contração muscular, sendo, portanto, mais leve ${ }^{18,20}$. Apenas no estudo de Niérat et al. ${ }^{5}$ esse parâmetro foi fixo, com 2,5 mA. Em um estudo o ajuste da intensidade não foi informado 20 .

A colocação dos eletrodos apresentou grande variabilidade entre os estudos de acordo com a área a ser estimulada e com o objetivo de cada autor. Vandermeeren et al. ${ }^{9}$ em seu estudo com ETCC, posicionou o eletrodo ativo na linha média sobre Fz no crânio e o eletrodo de referência extracefálico sobre a tíbia direita. Já no estudo de Azabou et al. ${ }^{10}$, essa colocação se deu para a A/ETCC com o ânodo sobre o córtex motor primário diafragmático esquerdo $(4 \mathrm{~cm}$ lateral à linha média e $1 \mathrm{~cm}$ anterior à linha binaural) e cátodo acima da órbita direita, e uma montagem contrária para a C/ETCC.

Um estudo com EET/EteCC, especificamente com uma estimulação elétrica transespinhal, dispôs os eletrodos de forma a colocar um sobre a linha média da parte posterior do pescoço (segmentos espinhais cervicais C3-C5), e o outro sobre a linha média da parte anterior do pescoço, logo abaixo do ângulo cervicomental, com a finalidade de aumentar a excitabilidade da via corticofrênica ${ }^{5}$.

As variáveis predominantes nos estudos foram: FR (frequência respiratória), VE (ventilação pulmonar ou 
ventilação-minuto), VC (volume corrente), PEmáx (pressão expiratória máxima), $V F_{1}$ (volume expiratório forçado no primeiro segundo), CVF (capacidade vital forçada) e VO2 (volume de oxigênio máximo); as quais, em geral, obtiveram melhoras com a terapia por estimulação elétrica. Todos os artigos com a EET apresentaram melhora de pelo menos uma variável de estudo. Já nos estudos com a ETCC, três estudos demonstram uma melhora em seus parâmetros ${ }^{11-13}$, outros três não evidenciaram alterações significativas em seus resultados ${ }^{9,14,15}$ e apenas um artigo apresentou um desfecho negativo da sua intervenção ${ }^{10}$.

No estudo de Azabou et al. ${ }^{10}$ que aplicou a corrente elétrica sobre $\mathrm{o}$ córtex motor primário diafragmático esquerdo em adultos jovens, tanto a ETCC anódica quanto a catódica diminuíram significativamente a amplitude do PEM do hemidiafragma, comparado com a ETCC simulada. Lee et al. ${ }^{13}$ encontraram uma associação positiva do exercício respiratório diafragmático com a A/ETCC, evidenciando que esta foi mais eficaz em melhorar a CVF e $\circ \operatorname{VEF}_{1}$ do que o exercício aliado à ETCC sham. Outro estudo encontrou que a A/ETCC aplicada no córtex pré-frontal combinada com exercício aeróbio submáximo posterior à estimulação aumentou o VO2 e o gasto de energia pelo menos por 30 minutos de recuperação ${ }^{12}$.

Para além disso, nos dois artigos que aplicaram a EET/TENS em pacientes pós cirurgia cardíaca, a estimulação elétrica desempenhou papel na melhora de parâmetros como 
da mecânica pulmonar da parede torácica, força muscular, bem como dos volumes e capacidades pulmonares ${ }^{16,17}$.

Niérat et al. ${ }^{5}$ estimularam segmentos espinhais a fim de explorar a possibilidade de alterar as projeções descendentes para motoneurônios frênicos, ou seja, modular comandos ventilatórios descendentes a partir da medula espinhal. Nesse estudo evidenciou-se que, curiosamente, a corrente elétrica catódica, e não a anódica, induziu um aumento persistente no volume corrente. Essa contradição é enfatizada pois a corrente catódica deveria exercer papel inibitório nas vias em questão e, portanto, diminuir o volume corrente. Ademais, encontraram que tanto a corrente anódica quanto a catódica produziram um aumento progressivo na amplitude do PEM diafragmático durante a estimulação que persistiu por pelo menos 15 minutos após o deslocamento da corrente.

Um estudo com pacientes com DPOC (estágio I ou II) revelou que a aplicação da corrente EET/Acu-TENS aumentou níveis de $V_{E F}$ e dispneia, mas sem efeitos significativos sobre a CVF7. Em contrapartida, um outro estudo com portadores de DPOC (estágio III e IV), dessa vez usando EET/EDET, destacou que após a intervenção não houve alteração na dispneia, mas uma interferência positiva na expansibilidade torácica e padrão respiratório desses pacientes ${ }^{20}$. 


\section{DISCUSSÃO}

A estimulação elétrica possui uma vasta aplicação terapêutica no organismo humano. Nesta revisão a maior parte dos estudos analisados utilizaram a ETT e o restante fez uso da ETCC.

De acordo com a análise dos estudos com ETCC observou-se a aplicação de variados protocolos experimentais, com uso da A/ETCC, C/ETCC e/ou ETCC sham. Em uma meta-análise publicada com ETCC observouse limitações recorrentes nos estudos devido à heterogeneidade significativa nos protocolos empregados pelo estudos ${ }^{21}$, o que pode ter acontecido nos estudos incluídos nesta revisão. Já nos estudos com EET utilizou-se diferentes tipos de corrente em seus métodos, como a EDET, TENS e EteCC. Assim, atenta-se que as diferenças metodológicas observadas podem contribuir para os diferentes desfechos encontrados posteriormente, a depender inclusive do tipo de corrente elétrica utilizada.

A grande maioria dos estudos com ETCC aplicou apenas uma sessão de estimulação elétrica para obtenção de resultados. Entretanto, estudiosos já afirmam que para melhores resultados da estimulação cerebral a área em questão deveria ser estimulada repetitivamente, supostamente devido a fatores cumulativos, inibitórios ou excitatórios ${ }^{22}$. Assim, sugere-se que sessões únicas de ETCC podem não fornecer informações confiáveis devido a multiplicidade de fatores externos que podem influenciar os resultados, particularmente com amostras pequenas. Nos 
estudos com EET apenas três estudos avaliaram os efeitos da estimulação sob maior prazo, dois com uso de 12 e um utilizando 30 sessões, podendo estabelecer resultados mais fidedignos ${ }^{6,18,20}$.

De acordo com os principais estudos e consensos de segurança de ETCC determina-se um tempo médio de aplicação de 20 minutos $^{23}$. Nos estudos apresentados essa também foi a média encontrada. Nesse sentido, a duração da estimulação torna-se um importante parâmetro da ETCC visto que, sob uma densidade de corrente constante, seu aumento poderá determinar os efeitos pós-estimulação. Ademais, conforme a duração de estimulação EET relatada, salienta-se que a aplicação de um campo elétrico com força e duração suficientes podem causar um rápido aumento na condutância elétrica das membranas biológicas e consequentes efeitos fisiológicos sobre os tecidos.

Sobre densidade de corrente ETCC, assim como nos estudos analisados, um estudo antecedente também estabeleceu a carga elétrica da corrente entre 1 ou $2 \mathrm{~mA}$, com a utilização de eletrodos de $35 \mathrm{~cm}^{224}$. Entretanto, valese destacar que um estudo prévio demonstrou que a estimulação de $2 \mathrm{~mA}$ induz um efeito comportamental maior em comparação com a de $1 \mathrm{~mA}^{25}$.

Houve uma grande variabilidade da intensidade de corrente nos estudos com EET. Isso se estabelece devido à heterogeneidade das várias formas de aplicação da EET, que, consequentemente, demandam diferentes protocolos de aplicação. Uma homogeneização desses protocolos poderia 
permitir a realização de uma posterior metanálise com maior acurácia de resultados e efeitos.

Além disso, sabe-se que o posicionamento do eletrodo é fundamental para determinar a direção e distribuição espacial do fluxo de corrente e, em última instância, a eficácia do tratamento. Na ETCC, a montagem dos eletrodos é programada com 0 intuito de um deles atingir especificamente determinadas áreas cerebrais e outro eletrodo de referência sobreposto em outro local para completar o circuito do fluxo da corrente, podendo ser bicefálica ou com eletrodo de referência extra-cefálico ${ }^{26}$. Nos estudos, esse posicionamento se revelou bastante diversificado.

Nos estudos com EET essa montagem varia mais, pois depende do objetivo da corrente aplicada nos tecidos fisiológicos. Dentre essas correntes e sua respectiva forma de aplicação, destaca-se a EteCC, posicionada próxima à região da coluna. Dado que o cérebro e a medula espinhal interagem por meio de várias projeções e que a estimulação da região cervical da medula sobre a coluna pode modular diferentes atividades supraespinhais, a EteCC emerge com inúmeras aplicações clínicas ${ }^{27}$, com efeitos neuromodulatórios nos circuitos espinhais relacionados às respostas motoras e sensoriais. Dessa forma, revela-se como uma aplicação da EET muito promissora no que se refere também à modulação de vias respiratórias e consequentes benefícios fisiológicos a pacientes com alguma disfunção respiratória. 
Diversas variáveis ventilatórias foram avaliadas nos estudos para determinação dos efeitos das correntes sobre a função músculo-ventilatória em humanos. Um resultado a ser destacado é que em um dos estudos analisados observou-se que tanto a ETCC anódica como a catódica induziram diminuição do PEM diafragmático ${ }^{10}$. Em contrapartida, a literatura aponta que a estimulação anódica deveria resultar em aumento da excitabilidade cortical e a catódica apresentar efeito inverso, reduzindo a excitabilidade. Esse achado evidencia que a neuromodulação com ETCC sobre áreas respiratórias cerebrais deve ser ainda melhor esclarecida. Para além disso, em outro estudo observou-se que a EET/EteCC catódica produziu um aumento persistente no volume corrente, e não a anódica ${ }^{5}$. Diante disso, propõe-se que mais pesquisas sejam realizadas com o objetivo específico de avaliar os efeitos das correntes anódicas e catódicas sobre a excitabilidade neuronal.

Ademais, sabe-se que os efeitos da ETCC nos resultados motores são altamente variáveis, variando inclusive entre as sessões e os indivíduos ${ }^{28}$. Estudos mais recentes têm verificado que os efeitos da corrente aplicada podem sofrer variação conforme o nível de excitabilidade cortical base, influenciando assim os resultados pesquisados ${ }^{29}$.

Os resultados com o uso da EET variaram conforme ao objetivo e a área a ser estimulada. Assim, a EET/TENS aplicada em pacientes pós cirurgia cardíaca melhorou parâmetros respiratórios ${ }^{16,17}$. Corroborando com este achado, um estudo de revisão, que avaliou o efeito da TENS 
no pós-cirúrgico cardíaco, já consolidou essa hipótese de que sua aplicação é eficaz não somente no alívio da dor, mas também na melhora da função musculo-ventilatória desses pacientes $^{30}$. Dessa forma, salienta-se que a TENS, comumente usada apenas para alívio da dor, pode desempenhar papel importante na melhoria funcional de alguns pacientes.

Em pacientes com DPOC, a EET foi eficaz para melhorar variáveis importantes, como VEF ${ }_{1}$, dispneia, expansibilidade torácica e padrão respiratório ${ }^{7,20}$. Sabe-se que a hiperinsuflação pulmonar característica desses pacientes ocasiona uma menor expansão da caixa torácica inferior, levando à diminuição da mobilidade costal, maior uso da musculatura acessória da respiração e, consequentemente, aumento da dispneia. Assim, uma melhora nesses parâmetros prevê uma melhora do quadro clínico obstrutivo do paciente com DPOC.

\section{CONCLUSÃO}

Diante desses resultados, evidencia-se que a natureza das correntes elétricas da ETCC e da EET são distintas. A ETCC baseia-se numa corrente com estimulação contínua de amperagem fixa entre 1 e $2 \mathrm{~mA}$ sobre pontos craniais específicos. Já a EET possui uma variedade de aplicações e de protocolos, com parâmetros que também se distinguem entre si de acordo com o objetivo e o tipo de corrente aplicada. 
Por conta dos diversos protocolos utilizados para a estimulação elétrica terapêutica, fica claro que o número de sessões, posicionamento dos eletrodos, duração da estimulação e a intensidade da mesma podem ter sido fatores importantes na determinação dos resultados encontrados.

No geral, os resultados da aplicação da EET se mostraram bastante promissores e satisfatórios na correlação com parâmetros da função musculoventilatória de humanos, como na CV, VC, VM, PImáx, PEmáx, dispneia, $V F_{1}$, entre outros. Já os achados relacionados à ETCC sobre funções respiratórias se revelaram pouco elucidados, com algumas discrepâncias que ainda precisam ser melhor esclarecidas, principalmente no que concerne aos efeitos excitatórios ou inibitórios da A/ETCC e C/ETCC. No entanto, essa técnica também tem se demonstrado promissora tendo em vista os avanços de pesquisas que envolvem seus efeitos sobre a ventilação.

Por conseguinte, sugere-se a realização de estudos futuros com melhor fundamentação, maior tamanho amostral, bem como com protocolos mais homogêneos a fim de um melhor esclarecimento dos efeitos fisiológicos da EET e da ETCC nos parâmetros musculoventilatórios em humanos, tanto a curto como a longo prazo. 


\section{REFERÊNCIAS}

1.Nitsche MA, Schauenburg A, Lang N, Liebetanz D, Exner C, Paulus $W$, et al. Facilitation of implicit motor learning by weak transcranial direct current stimulation of the primary motor cortex in the human. J Cogn Neurosci 2003;15:619-26.

https://doi.org/10.1162/089892903321662994

2.Stagg CJ, Nitsche MA. Physiological basis of transcranial direct current stimulation. Neuroscientist 2011;17:37-53.

https://doi.org/10.1177/1073858410386614

3.Raux M, Xie H, Similowski T, Koski L. Facilitatory conditioning of the supplementary motor area in humans enhances the corticophrenic responsiveness to transcranial magnetic stimulation. J Appl Physiol 2010;108:39-46. https://doi.org/10.1152/japplphysiol.91454.2008 4.DiMarco AF, Kowalski KE. Activation of inspiratory muscles via spinal cord stimulation. Respir Physiol Neurobiol 2013;189:438-49. https://doi.org/10.1016/j.resp.2013.06.001

5.Niérat MC, Similowski T, Lamy JC. Does trans-spinal direct current stimulation alter phrenic motoneurons and respiratory neuromechanical outputs in humans? A double-blind, Sham-controlled, Randomized, Crossover study. J Neurosci 2014;34:14420-9. https://doi.org/10.1523/JNEUROSCI.1288-14.2014

6. McLachlan AJ, McLean AN, Allan DB, Gollee H. Changes in pulmonary function measures following a passive abdominal functional electrical stimulation training program. J Spinal Cord Med 2013;36:97-103. https://doi.org/10.1179/2045772312Y.0000000031

7.Lau KSL, Jones AYM. A single session of Acu-TENS increases FEV1 and reduces dyspnoea in patients with chronic obstructive pulmonary disease: A randomised, placebo-controlled trial. Aust J Physiother 2008;54:179-84. http://dx.doi.org/10.1016/S0004-9514(08)70024-2 8.Tricco AC, Lillie E, Zarin W, O'Brien KK, Colquhoun H, Levac D, et al. PRISMA Extension for Scoping Reviews (PRISMAScR): Checklist and Explanation. Ann Intern Med 2018;169:467-73. https://doi.org/10.7326/M18-0850

9.Vandermeeren $Y$, Jamart J, Ossemann M. Effect of tDCS with an extracephalic reference electrode on cardio-respiratory and autonomic functions. BMC Neurosci 2010;11:38. https://doi.org/10.1186/14712202-11-38

10.Azabou E, Roche N, Sharshar T, Bussel B, Lofaso F, Petitjean M. Transcranial direct-current stimulation reduced the excitability of diaphragmatic corticospinal pathways whatever the polarity used. Respir Physiol Neurobiol 2013;159:183-7. https://doi.org/10.1016/j.resp.2013.07.024

11.Tomczak CR, Greidanus KR, Boliek CA. Modulation of chest wall intermuscular coherence: Effects of lung volume excursion and transcranial direct current stimulation. J Neurophysiol 2013;110:6807. https://doi.org/10.1152/jn.00723.2012

12. Montenegro R, Okano AH, Cunha FA, Fontes EB, Farinatti P. Does prefrontal cortex transcranial direct current stimulation influence the oxygen uptake at rest and post-exercise? Int J Sports Med 
2014;35:459-64. https://doi.org/10.1055/s-0033-1343452

13.Lee DJ, Lee YS, Kim HJ, Seo TH. The effects of exercise training using transcranial direct current stimulation (tDCS) on breathing in patients with chronic stroke patients. J Phys Ther Sci 2017;29:527-30. https://doi.org/10.1589/jpts.29.527

14.Baldari C, Buzzachera CF, Vitor-Costa M, Gabardo JM, Bernardes AG, Altimari LR, et al. Effects of transcranial direct current stimulation on psychophysiological responses to maximal incremental exercise test in recreational endurance runners. Front Psychol 2018;9:1-10. https://doi.org/10.3389/fpsyg.2018.01867

15.Park SB, Jun Sung D, Kim B, Kim SJ, Han JK. Transcranial Direct Current Stimulation of motor cortex enhances running performance. PLoS One 2019;14:1-11.

https://doi.org/10.1371/journal.pone.0211902

16.Cipriano G, Carvalho ACDC, Bernardelli GF, Peres PAT. Short-term transcutaneous electrical nerve stimulation after cardiac surgery: Effect on pain, pulmonary function and electrical muscle activity. Interact Cardiovasc Thorac Surg 2008;7:539-43. https://doi.org/10.1510/icvts.2007.168542

17.Gregorini C, Cipriano G, De Aquino LM, Rodrigues Branco JN, Bernardelli GF. Estimulação elétrica nervosa transcutânea de curta duração no pós-operatório de cirurgia cardíaca. Arq Bras Cardiol 2010;94:345-51. https://doi.org/10.1590/S0066782X2010000300011

18.Cancelliero KM, Ike D, Sampaio LMM, Santos VLA dos, Stirbulov R, Costa D. Estimulação diafragmática elétrica transcutânea (EDET) para fortalecimento muscular respiratório: estudo clínico controlado e randomizado. Fisioter Pesqui 2012;19:303-8. https://doi.org/10.1590/S1809-29502012000400002

19. Cancelliero-Gaiad KM, Ike D, Pantoni CBF, Mendes RG, Borghi-Silva A, Costa D. Acute effects of transcutaneous electrical diaphragmatic stimulation on respiratory pattern in COPD patients: cross-sectional and comparative clinical trial. Brazilian J Phys Ther 2013;17:547-55. https://doi.org/10.1590/S1413-35552012005000121

20.Santos IP, Cristina H, Uwamoto T, Amery TM, Maciel D, Rosa C, et al. Estimulação diafragmática pela corrente russa, cirtometria e padrão respiratório na DPOC. Salusvita (Bauru) 2015;34:265-75. https://secure.unisagrado.edu.br/static/biblioteca/salusvita/salusvita v34 n2 2015 art 06.pdf

21.Shiozawa $P$, Fregni $F$, Benseñor IM, Lotufo PA, Berlim MT, Daskalakis JZ, et al. Transcranial direct current stimulation for major depression: An updated systematic review and meta-analysis. Int J Neuropsychopharmacol 2014;17:1443-52.

https://doi.org/10.1017/S1461145714000418

22.Berlim MT, Neto VD, Turecki G. Estimulação transcraniana por corrente direta: uma alternativa promissora para o tratamento da depressão maior? Transcranial direct current stimulation : a promising alternative for the treatment of major depression ? Rev Bras Psiquiatr 2009;31(Supl I):34-8.

https://doi.org/10.1590/S1516- 
23.Bikson M, Datta A, Elwassif M. Establishing safety limits for transcranial direct current stimulation. Clin Neurophysiol 2009;120:1033-4. https://doi.org/10.1016/j.clinph.2009.03.018

24.Boggio PS, Rigonatti SP, Ribeiro RB, Myczkowski ML, Nitsche MA, Pascual-Leone $A$, et al. A randomized, double-blind clinical trial on the efficacy of cortical direct current stimulation for the treatment of major depression. Int J Neuropsychopharmacol 2008;11:249-54. https://doi.org/10.1017/S1461145707007833

25.Boggio PS, Bermpohl F, Vergara AO, Muniz AC, Nahas FH, Leme PB, et al. Go-no-go task performance improvement after anodal transcranial DC stimulation of the left dorsolateral prefrontal cortex in major depression. J Affect Disord 2007:101:91-8. https://doi.org/10.1016/j.jad.2006.10.026

26. Medeiros LF, Souza ICC, Vidor LP, Souza A, Deitos A, Volz MS, et al. Neurobiological effects of transcranial direct current stimulation: $A$ review. Front Psychiatr 2012;3:1-11. https://doi.org/10.3389/fpsyt.2012.00110

27. Cogiamanian F, Ardolino G, Vergari M, Ferrucci R, Ciocca M, Scelzo $\mathrm{E}$, et al. Transcutaneous Spinal Direct Current Stimulation. Front Psychiatr 2012;3:1-5. https://doi.org/10.3389/fpsyt.2012.00063

28.Chew T, Ho KA, Loo CK. Inter- and Intra-individual Variability in Response to Transcranial Direct Current Stimulation (tDCS) at Varying Current Intensities. Brain Stimulation 2015;8:1130-7. https://doi.org/10.1016/j.brs.2015.07.031

29.Bortoletto M, Pellicciari MC, Rodella C, Miniussi C. The interaction with task-induced activity is more important than polarization: A tDCS study. Brain Stimul 2015;8:269-76. http://dx.doi.org/10.1016/j.brs.2014.11.006 30. Oliveira APS, Cerqueira NS, Cordeiro AL, Oliveira AMS, Miranda ÍKSPB. Transcutaneous electrical nerve stimulation for pain treatment after cardiac surgery: A systematic review. Rev Pesqui Fisioter 2020;10:324-33. https://doi.org/10.17267/2238-2704rpf.v10i2.2808 